\title{
BENEFÍCIOS DO EXERCÍCIO AERÓBICO EM MULHERES NO PROCESSO DE EMAGRECIMENTO
}

Karina da Silva Faria

\begin{abstract}
RESUMO
A pesquisa consiste na análise dos diferentes tipos de treinamentos (treinamento aeróbico, treinamento resistido e treinamento concomitante) perante a diminuição do percentual de gordura corporal em mulheres. Seu objetivo reflexão sobre a importância do acompanhamento profissional na realização dos exercícios aeróbicos bem como elucidar os benefícios dos treinamentos para se obter os resultados esperados, por aquelas pessoas que desejam se beneficiar, movimentar o corpo engajando-se em um exercício físico que possibilite movimentar todos os músculos do corpo, podendo eliminar gordura e substituí-la por massa muscular bem como elucidar os fatores de risco associados à obesidade e sedentarismo por meio de uma revisão sistemática de artigos nacionais. Para tanto, foi realizada uma pesquisa nas bases de dados Medline, Scielo, Science direct, Pubmed, Capes, livros e revistas sobre o tema.
\end{abstract}

Palavras-chave:Exercícios aeróbicos. Perda de peso. Obesidade. Sedentarismo

\section{ABSTRACT}

\section{Benefits of the Aerobic Exercise in Women in the Slimming Process}

The research consists of the analysis of the different types of training (aerobic training, resistance training and concomitant training) in face of the decrease of body fat percentage in women. Its purpose is to reflect on the importance of professional accompaniment in the performance of aerobic exercises as well as elucidate the benefits of the training to obtain the expected results, for those people who wish to benefit, to move the body engaging in a physical exercise that makes it possible to move all The muscles of the body, being able to eliminate fat and replace it by muscular mass as well as elucidate the risk factors associated with obesity and sedentarism through a systematic review of national articles. In order to do so, a research was carried out in the databases Medline, Scielo, Science direct, Pubmed, Capes, books and magazines on the subject.

Keywords: Aerobic exercises. Weight loss. Obesity. Sedentarismo 


\section{INTRODUÇÃO}

A discussão sobre a efetividade da ginástica aeróbica sobre o índice de gordura corporal vem sendo feita no âmbito da Educação Física e da Medicina desde a década de 80, conforme Aquini (2004).

Para Repetto (1998 apud SALVE, 2006), possivelmente seja a doença mais antiga que se tem informação. Representações e esculturas em pedras já apresentavam mulheres obesas que foram deparadas em múmias egípcias.

Guedes (2007, p. 158) traz um esquema muito simples sobre o desenvolvimento da obesidade e suas consequências para o ser humano.

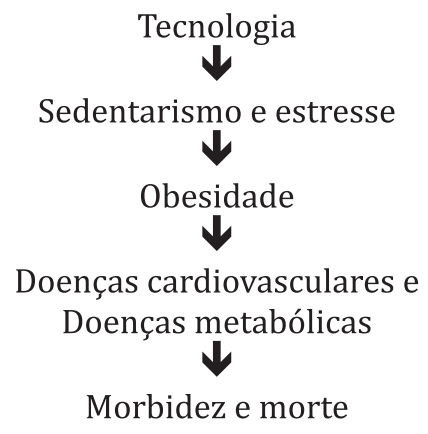

Conforme Maximiano (2012), as consequências para a saúde ligada à obesidade, existem fatores que vão desde condições que influenciam na qualidade de vidado ser humano, tais como problemas musculoesqueléticos, moléstias respiratórias, osteoartrite, até casos de infertilidade e ainda condições graves como as doenças coronarianas, diabetes tipo 2 e alguns tipos de câncer.

0 treinamento físico é uma atividade física esquematizada, estruturada e recorrente, que tem como objetivo melhorar ou conservar a saúde e a capacidade física, tendo em vista proporcionar benefícios. Dentre eles enfatiza-se a melhoria no condicionamento físico; a redução de peso; o aumento da força, coordenação e equilíbrio; a diminuição da inaptidão funcional e das moléstias físicas; melhoria do bem-estar além da redução da pressão arterial (PA) pós-exercício em analogia aos níveis pré-exercício.

Sob este prisma, Maximiano (2012) afirma que a reflexão sobre a importância da ginástica aeróbica a respeito do índice de gordura corporal tem sido feita no campo da Educação Física. Assim, a agregação de exercício físico e restrição alimentar (dieta) tem se revelado o procedimento mais eficiente para o tratamento e precaução dessa patologia, como também, tem indicado grande eficácia na sustentação do peso corporal a médio e longo prazos. 
Além disso, Santos (2010 p. 52) menciona que:

[...] uma dieta hipocalórica e baixa em gorduras, associada à prática de exercícios físicos regulares, constitui a base do tratamento para a diminuição dos riscos de doenças cardiovasculares em indivíduos obesos. Os indivíduos obesos são mais susceptíveis a riscos de lesões em articulações durante o exercício físico, por isso, muitas vezes a intensidade tem que ser baixa, apresentando um menor risco de lesões e diminuindo a sobrecarga músculo-articular, apesar de o trabalho ficar abaixo do ideal para o condicionamento aeróbico.

A partir do exposto anteriormente, a grande importância desta pesquisa é auxiliar na compreensão a obesidade, as alterações fisiológicas e as enfermidades ligadas a essa patologia, o processo de emagrecimento de obesos e a ajuda do exercício físico aeróbico para o emagrecimento.

Guedes (2007, p. 159) observa que o fator determinante para o emagrecimento é o balanço calórico negativo que apresenta três alternativas:

- diminuição da ingestão calórica;

- aumento do gasto calórico;

- diminuição da ingestão calórica e aumento do gasto calórico.

O interesse da pesquisadora pelo tema surgiu por ser profissional de Educação Física e perceber a necessidade de refletir sobre a questão da obesidade em mulheres de diferentes idades; bem como na literatura, o efeito dos exercícios aeróbios na oxidação de gordura corporal tendo em vista o expressivo aumento de obesos, principalmente entre mulheres.

Assim, o presente estudo propõe uma reflexão sobre a importância do acompanhamento profissional na realização dos exercícios aeróbicos destinados à redução da obesidade, especialmente em mulheres. Além disso, elucidar os benefícios dos treinamentos para se obter os resultados esperados, por aquelas pessoas que desejam se beneficiar, movimentar o corpo, engajando-se em um exercício físico que possibilite movimentar todos os músculos do corpo, podendo eliminar gordura e substituí-la por massa muscular.

0 presente estudo apresenta uma contextualização de obesidade e traz também um esboço acerca da relação entre obesidade e sedentarismo. 
Efetua uma reflexão sobre o exercício aeróbico, evidenciando o que é e sua importância na diminuição do percentual de gordura corporal e redução do peso, ajudando ainda na melhora da aptidão cardiovascular.

Reflete-se ainda sobre a necessidade de um acompanhamento do profissional habilitado, pois uma atividade bem planejada e orientada adequadamente proporciona melhores resultados.

0 estudo foi relevante para a pesquisadora e espera-se que sirva de incentivo a fim de que outros estudiosos se interessem pelo assunto e possam também elucidar dúvidas e apresentar novos aspectos referentes ao tema, o que irá enriquecer a literatura atual.

\section{METODOLOGIA}

A metodologia empregada para a efetivação deste estudo foi uma revisão de literatura realizada por meio das bases de dados do Google Acadêmico e Scielo, e também foram utilizados livros, revistas e periódicos. Para localizar os artigos foram usadas as seguintes palavras-chave: obesidade, obesidade e emagrecimento, sobrepeso e obesidade, exercício físicos aeróbicos, métodos de emagrecimento, alterações fisiológicas associadas à obesidade, exercícios aeróbicos e obesidade, exercícios resistidos e obesidade.

Foram utilizadas obras no idioma português, publicadas nos anos de 2001 a 2016.

\section{FUNDAMENTAÇÃO TEÓRICA}

\section{OBESIDADE: CONTEXTUALIZAÇÃO}

0 mundo moderno tem presenciado uma mudança nos padrões alimentares e nutricionais. Coqueiro et. al. (2008) comentam que fundamentalmente no Brasil a desnutrição foi superada pela obesidade desde a década de 1990.

Segundo Castro citado por Coqueiro et al.(2008), depois de vários estudos epidemiológicos se confirmou que a incidência do sobrepeso está vinculada a qualidade da alimentação, as atividades físicas diárias e ao espaço físico e social.

Conforme Reis Filho et al. (2008), a obesidade pode ser considerada quanto à procedência, a distribuição de gordura, ao desenvolvimento do tecido adiposo e quanto à morbidade. Ainda de acordo com os autores, é considerado "[...] obeso a pessoa que apresenta IMC com gordura corporal acima de $25 \%$, para os homens e de $35 \%$ ou mais para as mulheres.". 
Para Francischi (2001, p. 18), “a obesidade é um distúrbio do metabolismo energético, com um armazenamento em forma de triglicérides."

Conforme REIS, Filho (2000, p.13):

A obesidade é uma doença considerada como a nova epidemia do fim do século XX e começo do século XXI. É analisada como mais grave que a desnutrição, por isso necessita ser abordada por especialistas multidisciplinares competentes.

Atualmente, a obesidade tem sido ponderada como um problema de saúde pública, que possui diferentes causas. Para Costa et al (2003), ela assinala-se como uma anomalia metabólica ocasionada pela ingestão excessiva de calorias, o que causa um acúmulo de triglicerídeos nas células gordurosas, disseminados pelo corpo.

Percebe-se de acordo com Coqueiro et al. (2008) que a obesidade, é uma doença de procedência multifatorial, que pode causar consequências traumáticas, sobretudo ao adolescente, sendo indispensável a interferência dos pais, que através instruções recebidas de profissionais habilitados, podem intervir no tratamento, e assim auxilia-los nessa fase, o que é imprescindível. Assim, uma pessoa desde a infância, até atingir sua fase adulta poderá correr sérios riscos para doenças do coração como: hipertensão arterial, colesterol e triglicerídeos elevados entre outros.

Pode-se notar que a obesidade é um procedimento gradativo, em que a ingestão excessiva de alimentos e um pequeno gasto calórico, podem constituir como fatores para que a obesidade aconteça. Assim para Canevali (2011, p. 31), "A obesidade é uma doença complexa com etiologia multifatorial e consequências metabólicas heterogêneas. Ocorre quando há um desequilíbrio crônico entre energia ingerida e a consumida."

Diante desta realidade, sob a ótica de Reis Filho et al. (2008, p. 36), estudos e pesquisas mostram complicações com o exagero de peso, e nota-se que a obesidade é uma enfermidade de complexo controle, com elevadas percentagens de insucessos, podendo ainda apresentar em seu progresso, sérias repercussões no organismo e psicossociais. "O obeso é perseguido, agredido e marginalizado posteriormente, afastando-se do convívio social e esportivo, agravando o processo." (REIS FILHO, 2008, p. 36) 


\subsection{Obesidade e sedentarismo}

De acordo com Matsudo (2007) vive-se em um mundo invadido por duas endemias: a de sedentarismo e a de obesidade. Comenta a autora que ambas correspondem a implicações de diversos fatores. Um dos fatores causadores pela maior prevalência de excesso de peso e obesidade é o sedentarismo, ou seja, a ausência da prática de atividade física regular e obesidade há muito é analisada como fator de risco cardiovascular. Porém, só muito depois, passou a ser considerada a inatividade física como fator de risco independente.

Segundo Nahas (2006) com o progresso da tecnologia a sociedade contemporânea está cada vez mais adotando os confortos e comodismos, o que leva todas as pessoas a terem uma existência sedentária, rotina esta, que é analisada como um fator de risco para o surgimento de doenças cardiovasculares. Já que a possibilidade de ocorrer um infarto é duplamente maior em sedentários quando confrontados com aqueles regularmente ativos.

O sedentarismo constitui um atributo primário da maior parte das mulheres com peso corpóreo acima do normal de acordo com Matsudo (2007). Este comportamento sedentário observa a autora, "[...] parece ser um dos principais agentes causadores do excesso de gordura, como também, em contrapartida, a obesidade parece conduzir o indivíduo a uma diminuição dos níveis de atividade física." (MATSUD0, 2007, p. 104).

A inatividade física e a alimentação inadequada conforme Melo et al. (2008) ocasionam balanço energético positivo, o que exprime acréscimo do peso corporal. A complexidade da regulação do peso corporal concebe um dos maiores desafios para a compreensão da etiologia, terapêutica e precaução da obesidade. A inatividade física e o excesso de peso corporal estão intimamente coligadas com o risco de aumentar os casos de enfermidades cardiovasculares constituindo-se nos fatores de risco mais expressivos.

A Organização Pan-Americana de Saúde (OPAS, 2003) adverte que a atividade física é um benefício na vida cotidiana do indivíduo, sendo que com sua prática regular é possível melhorar os hábitos alimentares, evitar o tabagismo, reduzir a violência e aprimorar a capacidade do indivíduo às relações sociais.

\section{EXERCÍCIO AERÓBICO E ANAERÓBICO}

Matsudo (2007) explica que existem dois tipos de Exercícios Físicos, os aeróbios e anaeróbios. Tanto oexercício aeróbio quanto o anaeróbio está atrelado ao tipo de metabolismo energético que será usado. 0 autor afirma que isto não está associado aos resultados saudáveis dos exercícios. Ambos os tipos de treinamentos podem ser de amplitude leve, moderada ou forte. 
$\mathrm{Na}$ realização de exercício aeróbico o oxigênio atua como fonte de queima das substâncias que produzirão a energia conduzida para o músculo em agilidade. É' um exercício de extensa duração, serve para melhorar as articulações gastas. Excita a função dos sistemas cardinal e vascular e ainda o metabolismo, visto que acrescenta a capacidade cardíaca e pulmonar para prover de energia o músculo a partir do gasto do oxigênio, razão do nome aeróbico.

Saba (2003) assim considera exercício aeróbico:

“[...] exercício aeróbico todo esforço que possa ser mantido por um longo período de tempo. Costumam ser, portanto, esforços de intensidade leve ou moderada, definindo-se essa intensidade de acordo com a aptidão individual."] (Saba, 2003 p.106).

Desta forma, para o autor mencionado a caminhada, a corrida, a natação, o ciclismo e a ginástica aeróbica são exercícios normalmente analisados como aeróbicos por causa do modo de exigência cardíaca e muscular que geram, sendo aceitável a sua prática delongada.

No que diz respeito ao objetivo de diminuição de peso, Matsudo (2007) assegura que é amainar a porcentagem de gordura corporal, evidencia-se o exercício aeróbico por causar a movimentação e utilização dos ácidos graxos como principal fonte de energia.

De acordo com Matsudo (2007), as atividades aeróbicas ajudam na melhora da aptidão cardiovascular e trabalham na prevenção da arteriosclerose e hipertensão. (BEAN, 1999, p.64) observa que:

[...] o treinamento aeróbico fornece numerosos benefícios para os pacientes de treinamento de força como:

- reduz a gordura corporal e mantem baixas as porcentagens de gordura corporal;

-aumenta a capacidade do corpo de queimar gorduras durante os exercícios e nos períodos de descanso.

- melhora a composição corporal;

- melhora o bem-estar cardiovascular (estamina);

- reduz o estresse e a ansiedade;

- melhora a confiança, a autoestima e o humor;

- reduz a pressão sanguínea, o colesterol e o risco de problemas cardíacos. 
Guedes et al. (2008, p. 214) enumeram sugestões fundamentais para as prescrições e as orientações dos treinamentos aeróbicos voltados para o controle de peso corporal tais como:

-frequência: de 3 a 5 sessões por semana, em dias alternados, quando possível;

-intensidade: $40 \%$ a $65 \%$ da frequência cardíaca de reserva com base na idade e na frequência cardíaca de repouso. Esforços de intensidade mais baixa também podem produzir importantes benefícios em indivíduos de menor condição aeróbica;

-duração: de 30 a 60 minutos de maneira contínua a cada sessão;

-tipo de atividade: preferencialmente caminhada, corrida, ciclismo e natação, ou seja, exercícios físicos que envolvem grandes grupos musculares.

Para Santos (2010),é importante que haja um tempo de adaptação, desta forma, o treinamento igualmente necessita ser adequado, como exemplo, para sujeitos com menor resistência precisa-se praticar uma caminhada comum tempo maior de constância e menor amplitude ajustando gradualmente conforme com seu desenvolvimento.

Para Nahas (2006),os exercícios anaeróbios são aqueles que apresentam intensidade e de curta duração sem a participação do oxigênio para aquisição de potência. Correr em alta velocidade e levantar pesos são exemplos de atividades anaeróbias. Nahas (2006) esclarece que o vocábulo anaeróbico faz referência a um determinado tipo de bactéria (anaeróbica), capaz de produzir energia, sem a presença de oxigênio. As adequações indispensáveis acontecem nos próprios músculos proporcionando benefícios localizados. Esse é o modelo utilizado nos exercícios com peso.São exemplos de exercícios anaeróbios os exercícios de velocidade com ou sem carga, de curta duração e alta intensidade, como a corrida de cem metros rasos, os saltos, o arremesso de peso.

Exercícios de força ou exercícios resistidos, como a musculação também são considerados como exercício anaeróbio. A esse respeito Santos (2010, p. 27) pondera que:

A fisiologia do exercício anaeróbio acontece através de dois sistemas, o primeiro denominado Sistema Anaeróbio Alático, pela combinação de ATP (Adenosina Trifosfato) e CP (Creatina Fosfato), gera e armazena energia no músculo. E o Sistema Anaeróbico Lático ou 
Glicólise Anaeróbica, o qual o processa a substancia alimentar denominada carboidrato, em ácido lático, que por sua vez será utilizado nos músculos em forma de glicogênio. Dentre as funções do exercício anaeróbico, pode-se destacar o estimulo a resistência, densidade do osso e flexibilidade.

Segundo Añez; Petroski (2002), na organização da prescrição de exercício como fim de controle da gordura corporal, sempre se deve- atender às quatro variáveis fundamentais dos exercícios aeróbicos: frequência, intensidade, duração e tipo. Uma vez que o aluno tenha entrado num programa adequado de exercícios aeróbicos, pode-se acrescentar ao programa, levantamento com pesos. Entretanto, o propósito inicial deve ser o desenvolvimento do volume do exercício e do consumo calórico, que pode ser melhor alcançado pela concretização de exercício aeróbico. 0 treino de resistência pode terminar num pequeno aumento da massa corporal magra, o que aumentará o consumo energético pelo funcionamento do metabolismo de repouso, mas seu resultado é comparativamente baixo.

Souza (2012) comenta que a duração média deste tipo de exercício deve ser de 60 minutos, sendo frequência mínima de duas vezes por semana. Para obter o resultado esperado, é necessário se adaptar a rotina de exercícios, respeitar o limite do corpo, sem exagerar no peso, bem como dar pausas para o descanso e recuperação muscular.

\subsection{Benefícios do treinamento aeróbico e importância da atuação do profissional de Educação Física.}

A procura pela atividade física, que não traz um fim em si mesmo e, mais benefícios proporcionados por eles, de acordo com Barbosa (2003), têm suscitado numerosos estudos os quais tem assinalado destaques científicos que sustentam melhoramentos relacionados a esta, como cuidado e reabilitação de moléstias cardiovasculares, câncer, síndrome metabólica, osteoporose entre outras. Tais benefícios de acordo com a ACMS (2003) ocasionam a melhora das aptidões funcionais, a complementação de tratamentos clínicos e fisioterápicos e o incremento de capacidades cognitivas, sociais e psíquicas.

Alcântara (2004) afirma que o desempenho do Profissional de Educação Física consiste em promover um modo de vida benéfico por meio da atividade física, por ser um meio eficaz para a construção grupal da qualidade de vida. Além disso, é também uma função deste profissional planejar e realizar atividades físicas, tendo em vista a atividade como terapia e como incentivo à adesão do 
exercício por meio da autoestima, consciência corporal, autonomia no viver e em seu processo de controle de peso.

O Profissional de Educação Física necessita articular as redes sociais, como o propósito de organizar e planejar atividades que promovam a melhoria do modo de vida saudável, até mesmo a partir de projetos. Sobre este aspecto Dias (2007) relata a atividade física dirigida para diabéticos usada como um procedimento de precaução e tratamento da Diabetes Mellitus (DM) e que auxilia na prevenção e controle de diferentes doenças como hipertensão e obesidade, e isto resulta uma melhor condição de vida para a população e reduz os gastos que a saúde pública possa ter.

Matsudo (2007) evidencia que é grande a importância da profissional de Educação Física, nada melhor do que colocar a atividade orientada e o profissional de que dela se ocupa como tendo um papel muito central no programa de tratamento das diversas doenças.

Para Reis Filho (2008) na opção pelo melhor exercício é importante levar em consideração 'aquele que é prazeroso'. Nesse aspecto o Profissional de Educação Física tem uma capacitação e treino específico, o que o torna capaz de ajudar na sugestão, escolha apropriada e estímulo à prática de exercícios por meio de uma prescrição polida e personalizada, não limitando o treineiro a restringir-se unicamente à caminhada, que é comumente recomendada e com certeza muitas vezes uma boa escolha, mas que não é a única.

\section{FISIOLOGIA DO EXERCÍCIO}

0 termo fisiologia, de acordo com Greco (2011), origina-se do grego "physis" que quer dizer natureza, desempenho ou funcionamento e "logos" que por sua vez significa palavra ou estudo. Deste modo, a Fisiologia distingue-se como aparte da Biologia que explora os múltiplos desempenhos mecânicos, físicos e bioquímicos dos seres vivos. Ela emprega os conceitos da física e da química para esclarecer como acontecem as funções fundamentais dos diversos organismos e suas adequações frente aos estímulos do meio ambiente.

Nessa circunstância, explica Plowman(2009), a Fisiologia do Exercício que também denominada como Fisiologia do Esforço ou da Atividade Física, é uma ciência proveniente da disciplina mãe Fisiologia, que analisa como as funções do organismo respondem e se ajustam ao estresse imposto pelo exercício físico. Em outras palavras, a Fisiologia do Exercício observa os resultados agudos e recorrentes do exercício físico sobre a estrutura e a função dos diferentes aparelhos orgânicos. 
Em complementação, a Fisiologia do Exercício indaga também a influência mútua entre os diversos resultados do exercício físico e a influência dos estressores ambientais.

Conforme Souza et al (2012) percebe-se como decorrências agudas, denominadas de "respostas", as alterações resultantes do desempenho de uma sessão de exercícios. Tais respostas são fracionadas em resultados observados no decorrer do exercício e respostas analisadas depois o exercício. As últimas podem ainda ser divididas em respostas imediatas, que acontecem nas uma ou duas horas posteriores ao exercício, e tardias, que são analisadas no período de 24 horas depois do exercício. " No que se refere aos resultados recorrentes, cognominados 'adaptações', eles correspondem às transformações estruturais e funcionais resultantes de um período delongado de treinamento físico regular."(SOUZA,2012 p. 13)

Silva (2010) comenta que é conveniente observar, no entanto, que investigações utilizando o exercício físico e análise das respostas fisiológicas são realizadas também por diversos estudiosos da fisiologia básica, expõe ainda que é importante relacionar as diferenças de tais pesquisas conduzindo-as para as análises especiais, de natureza mais aplicada, em Fisiologia do Exercício. Para Astrand (2006) na fisiologia básica, o exercício é utilizado como um estressor ao organismo, assim como poderia ser utilizado um estresse térmico, psicológico ou qualquer outro.

Wilmore (2010) ressalta que o mais importante é colocar o organismo em uma condição de inconstância e averiguar suas respostas. Fica evidente que o exercício físico é aproveitado como um elemento de investigação científica. Porém, para os pesquisadores da Fisiologia do Exercício, as informações da fisiologia básica são empregadas para esclarecer as respostas dos seres humanos ao exercício. 0 exercício passa a ser "[..] o ponto principal da análise, ele é o objeto de estudo propriamente dito, é a finalidade da pesquisa." (WILMORE, 2010, p. 82)

Plowman et al. (2010) subdividem a Fisiologia do Exercício edefinem a Fisiologia do Esporte como uma ciência do conhecimento que concentra os conceitos da Fisiologia do Exercício na preparação e coordenação de elementos, processos e programas de treinamento volvidos, nomeadamente, para o avanço do desempenho físico-esportivo de atletas. Neste aspecto, a nomenclatura Fisiologia do Exercício deverá ser empregada para representar o emprego das informações fisiológicos às situações da Educação Física e do Esporte.

Segundo TAYLOR (apud LEITE, 1990, p.4): 
[...] o homem adota a prática de exercícios físicos por nove motivos: busca do lazer, estabilidade emocional, desenvolvimento intelectual, consciência estética, competência social, desenvolvimentos morais, autorrealização, desenvolvimento das capacidades motoras e desenvolvimento físico-orgânico.

\subsection{Aspectos da Fisiologia do Exercício Feminino}

Segundo Cordeiro (2011)pode-se notar diferenças na fisiologia entre homens e mulheres quanto ao exercício presente antes da puberdade e acrescem no decorrer da adolescência e a vida adulta. Estas sucedem basicamente em função de tamanho e composição corporal.

Ainda parafraseando o autor citado, comumente os homens têm maior massa muscular em termos absolutos e relativos (conforme o peso corporal total), enquanto que as mulheres apresentam maior percentagem de gordura corporal, o que decorre numa menor eficácia termo regulatória nos exercícios em espaços quentes. Apesar da composição de fibras musculares ser parecida nos homens e mulheres, o volume de toda fibra, seja do tipo I ou II, é maior em relação aos homens. Tais peculiares atribuem maior energia e endurance muscular aos homens.

Graves e Franklin (2006)observam, em relação ao exercício de força para mulheres, evidenciam as respostas fisiológicas principais como celular, tipo de fibra, sistema de proteínas e adequações neuromusculares. Estas respostas fisiológicas são iguais em homens e mulheres no exercício intenso, porém a mulher tem menos massa muscular, e suas variações são mais manifestas nas fibras do tipo I em correlação com as fibras IIa e IIb, sendo que nos homens existem mais alterações nas fibras do tipo IIa que as fibras do tipo I.

Durante exercícios aeróbicos de acordo com Ide et al (2010, p. 91):

[...] observa-se menor consumo máximo de oxigênio em mulheres em comparação com os homens, sendo que o principal mecanismo hemodinâmico envolvido é o menor débito cardíaco decorrente de menor volume sistólico. Esta característica, por sua vez, é consequente à menor 
massa e volume ventriculares em mulheres, seja do ponto de vista absoluto ou relativo ao peso corporal total. Além disso, a capacidade de transporte de oxigênio (devido a um nível médio de hemoglobina inferior decorrente das menstruações) é menor nas mulheres. Estes fatores em conjunto fazem com que o desempenho desportivo seja 6 a $15 \%$ menor nas mulheres em comparação com os homens, embora a capacidade de adaptação ao treinamento seja semelhante.

Astrand (2006) evidencia que a performance masculina na realização das atividades competitivas é geralmente melhor que o feminino. Não obstante, alguns estudos recomendam que em corridas de grande duração tenha a probabilidade de que o desempenho de mulheres chegue a ser futuramente similar ou até mesmo melhor que o masculino.

Ide et al (2010) comenta que a mais acentuada naquelas é um maior dispêndio de $\mathrm{O}_{2}$ no decorrer da caminhada ou corrida de amplitude próxima do máximo (menor eficiência mecânica), o que acarreta menor reserva metabólica e fadiga mais precoce. Apesar das respostas fisiológicas ao exercício em garotas antes da puberdade apresentarem desempenho equivalente ao perfil de mulheres adultas, existem diferenças que necessitam ser consideradas.

Assim, conhecer bem os exercícios, como influenciam no metabolismo e respostas fisiológicas de amoldamento para realizá-los, são essenciais para alcançar um efeito positivo dentro de um planejamento de treinamentos direcionados para a perda de peso é muito importante para um resultado almejado.

\section{CONSIDERAÇÕES FINAIS}

A forma física e a boa saúde não são sinônimos, mas complementares entre si. Enquanto uma boa saúde significa simplesmente a ausência de doenças, a forma física pressupõe energia suficiente para buscar as recompensas da vida e não depender fisicamente de outras pessoas.

Os estudos realizados apontaram a importância dos programas de exercício físico aeróbico para o emagrecimento e à saúde. Exercícios aeróbicos são os mais aconselhados; entretanto, a adição de exercícios resistidos pode ser benéfico na ampliação de força e precaução da perda da massa livre de gordura. Cuidados específicos na ocasião da deliberação do volume-intensidade do exercício são 
importantes, pois os obesos não são muito afetos à prática de exercícios físicos, sendo apropriada uma sugestão de programas de exercícios individualizados.

As pessoas estão cada vez mais preocupadas com as questões estéticas, onde há uma inquietação em seguir novos hábitos saudáveis que originam a melhora na Qualidade de Vida. 0 profissional de Educação Física é a pessoa que está presente na vida das pessoas desde cedo, assim, tem grande ingerência nas alternativas do modo de vida das pessoas.

Para dar início a qualquer tipo de Atividade Física é influenciável pela mídia, pela família e amigos na procura para alcançar seus objetivos tanto estéticos como de saúde. A adoção à rotina de praticar Exercícios Físicos com regularidade e bem dirigidos por um professor de Educação Física oferece condição para enfrentar a hodierna vida do dia-a-dia com jornada trabalho e os trabalhos do cotidiano. Assim sendo, a prática regular de Exercício Físico proporciona uma situação de bem-estar biopsicossocial donde se consegue uma saúde estável sem presságios e isso assinala a promoção da Qualidade de Vida.

Os exercícios físicos evidenciam melhoras na estética e saúde;logo, ocasionam grandes benefícios a incômodos físicos como também minoram graves doenças. A realização dessa pesquisa contribuiu para o crescimento o profissional da pesquisadora, quando foi possível compreender melhor o mundo da academia na qual está inserida, sobretudo no que se refere ao treinamento feminino e a tão sonhada procura pelo corpo ideal. É gratificante poder auxiliar as minhas "parceiras de treino" a uma vida mais saudável e por que não dizer mais fácil, simples na busca por respostas melhores para meu corpo.

Pode-se perceber que os efeitos do exercício vão além da perda de peso, agenciando também a redução do percentual de gordura, o acréscimo da massa magra, melhora da capacidade aeróbia, além de proporcionar benefícios relacionados aos fatores de riscos cardiovasculares, adjuntos à síndrome metabólica. É importante lembrar que os estudos recomendam o treinamento aeróbio realizado simultaneamente com o treinamento de força que proporciona os melhores resultados em indivíduos obesos. Entretanto ainda é necessário elucidar a doseresposta para as diversas modalidades de exercício para aperfeiçoá-la no processo de emagrecimento.

Este estudo abre portas para outras pesquisas que tenham como objetivo a redução de gordura corporal, utilizando os treinamentos de força, aeróbico, concomitante para esse público muito exigente, "nós, mulheres". 


\section{CONCLUSÃo}

A forma física e a boa saúde não são sinônimos, mas complementares entre si. Enquanto uma boa saúde significa simplesmente a ausência de doenças, a forma física pressupõe energia suficiente para buscar as recompensas da vida e não depender fisicamente de outras pessoas.

Os estudos realizados apontaram a importância dos programas de exercício físico aeróbico para o emagrecimento e à saúde. Exercícios aeróbicos são os mais aconselhados; entretanto, a adição de exercícios resistidos pode ser benéfico na ampliação de força e precaução da perda da massa livre de gordura. Cuidados específicos na ocasião da deliberação do volume-intensidade do exercício são importantes, pois os obesos não são muito afetos à prática de exercícios físicos, sendo apropriada uma sugestão de programas de exercícios individualizados.

As pessoas estão cada vez mais preocupadas com as questões estéticas, onde há uma inquietação em seguir novos hábitos saudáveis que originam a melhora na Qualidade de Vida. 0 profissional de Educação Física é a pessoa que está presente na vida das pessoas desde cedo, assim, tem grande ingerência nas alternativas do modo de vida das pessoas.

Para dar início a qualquer tipo de Atividade Física é influenciável pela mídia, pela família e amigos na procura para alcançar seus objetivos tanto estéticos como de saúde. A adoção à rotina de praticar Exercícios Físicos com regularidade e bem dirigidos por um professor de Educação Física oferece condição para enfrentar a hodierna vida do dia-a-dia com jornada trabalho e os trabalhos do cotidiano. Assim sendo, a prática regular de Exercício Físico proporciona uma situação de bem-estar biopsicossocial donde se consegue uma saúde estável sem presságios e isso assinala a promoção da Qualidade de Vida.

Os exercícios físicos evidenciam melhoras na estética e saúde logo, ocasiona grandes benefícios a incômodos físicos e minoram graves doenças também. A realização dessa pesquisa fez a pesquisadora crescer muito como profissional. Foi possível compreender melhor o mundo da academia na qual estou inserida, sobretudo no que se refere ao treinamento feminino e a tão sonhada procura pelo corpo ideal, é gratificante poder auxiliar as minhas "parceiras de treino" a ter uma vida mais saudável e por que não dizer mais fácil, simples na busca por respostas melhores ao meu corpo.

Pode-se perceber que os efeitos do exercício vão além da perda de peso, agenciando também a redução do percentual de gordura, o acréscimo da massa magra, melhora da capacidade aeróbia, além de proporcionar benefícios relacionados aos fatores de riscos cardiovasculares adjuntos à síndrome metabólica. 
É importante lembrar que os estudos recomendam que o treinamento aeróbio realizado simultaneamente com o treinamento de força proporciona os melhores resultados em indivíduos obesos. Entretanto ainda é necessário elucidar a doseresposta para as diversas modalidades de exercício para aperfeiçoar o papel do exercício no processo de emagrecimento

Este estudo abre portas para outras pesquisas que tenham como objetivo a redução de gordura corporal, utilizando os treinamentos de força, aeróbico e concomitante para esse público muito exigente "nós, mulheres".

\section{REFERÊNCIAS}

ALCÁNTARA, F. C. Estudo Bibliográfico Sobre o Processo Histórico de Atuação do Educador Físico e da sua Inserção na EstratégiaSaúde da Família do Município de Sobral-CE. 2004. 65f. Monografia (Especialização com Caráter de Residência em Saúde da Família) - Universidade Estadual Vale do Acaraú. Acaraú, 2004.

PESCATELLO, L. S.; ARENA, R.; RIEBE, D.; Thompson, P. D. Diretrizes do ACMS para os testes de esforço e sua prescrição. 6. ed. Rio de Janeiro: Guanabara Koogan, 2003.

ANEZ, C. R. R.; PETROSKI, E. L. 0 exercício físico no controle do sobrepeso corporal e da obesidade. Revista Digital, Buenos Aires, ano 8, n. 52, set. 2002. Disponível em: <http://www.efdeportes.com/>. Acesso em:14/12/2016.

AQUINI, L. F. M. P. A efetividade de um programa de treinamento em ginástica aeróbica sobre o percentual de gordura corporal em jovens do sexo feminino. EFDeportes.com, ano. 10, n. 69, p. 1-1, fev. 2004. Disponível em: < http://www.efdeportes.com/efd69/ga.htm>. Acesso em 18 dez. 2016.

ASTRAND, P. O. et al. Tratado de fisiologia do trabalho. 4. ed. Porto Alegre: Artmed, 2006.

BARBORSA, J. A. S.; BANFOFF, A. D. P. Estudo sobre o nível de participação, num programa de atividade física e saúde e suas relações com as doenças crônicas não transmissíveis. Movimento \& Percepção, Espirito Santo do Pinhal,v. 9, n. 12, p. 197 220, jan./jun. 2008.

BEAN, A. 0 guia completo de treinamento de força. 1. ed. São Paulo: Brasileira, 1999. 
CANEVALI JUNIOR, L. C. et al. Exercício, Emagrecimento e Intensidade do treinamento: aspectos fisiológicos e metodológicos. São Paulo: Phorte, 2011.

COQUEIRO, R. S. et al.. Fatores associados ao Sobrepeso em Adultos acompanhados por uma Unidade de Saúde da Família. Revista Brasileira de Cineantropometria e Desempenho Humano, Salvador, v. 10, n. 2, p. 149-154, set. 2008.

CAVALCANT, A. P. R. et al. Crenças e influências sobre dietas de emagrecimento entre obesos de baixa renda. Ciência \& Saúde Coletiva, v. 12, n. 6, p. 1567-1574, 2007.

DIAS, J. A. et al.A importância da execução de atividade física orientada: uma alternativa para o controle de doença crônica na atenção primária. EFDeportes.com, Buenos Aires, ano. 12, n. 114, nov. 2007 . Dis ponível e m: <http://www.efdeportes.com/efd114/a-importancia-da-execucao-de-atividadefisica-orientada.htm $\geq$ Acesso em:, 15 dezembro 2016:

FRANCISCHI, R. P. et al. Exercício, comportamento alimentar e obesidade: revisão dos efeitos sobre a composição corporal e parâmetros metabólicos. Revista Paulistana de Educação Física, São Paulo, v. 15, n. 2 p. 117-40, jul./dez. 2001. Disponível em: <http://www.portalsaudebrasil.com/ artigospsb/obes166.pdf>. Acesso em: $14 \mathrm{dez}$. 2016.

GUEDES, D. P. et al. Impacto da prática habitual de atividade física no perfil lipídico de adultos.Arq Bras Endocrinol Metab., v. 51, n. 1, p. 72-78, fev. 2007.

GRECO, C. C. Aspectos fisiológicos e técnicos da natação. In: RANGEL, I. C. A.; DARIDO, S. C. (Org.). Educação física no ensino superior. Rio de Janeiro: Guanabara Koogan, 2011.

IDE, B. N.; LOPES, C. R.; SARRAIPA, M. F. Fisiologia do treinamento esportivo: força, potência, velocidade, resistência, periodização e habilidades psicológicas. São Paulo: Phorte, 2010.

MATSUDO, S. M.; MATSUDO, V. K. R. Atividade física e obesidade, prevenção e tratamento. São Paulo: Atheneu, 2007.

FOUREAUX, G.; CASTRO PINTO, K. M.; DÂMASO, A. Efeito do consumo excessivo de oxigênio após exercício e da taxa metabólica de repouso no gasto energético.Rev. Bras. Med. Esporte, Niterói, v. 12, n. 6, p. 393-398. nov./dez. 2006. 
MELO, C. M.; TIRAPEGUI J.; RIBEIRO, S. M. L. Gasto energético corporal: conceitos, formas de avaliação e sua relação com a obesidade. Arq. Bras. Endocrinol. Metab., São Paulo, v. 52, n. 3, p. 452-464, abr. 2008.

NAHAS, M. V. Atividade física, saúde e qualidade de vida: conceitos e sugestões para um estilo de vida ativo. 4. ed. Londrina: Mediograf, 2006.

ORGANIZAÇÃO PAN-AMERICANA DA SAÚDE. Doenças crônico-degenerativas e obesidade: estratégia mundial sobre alimentação saudável, atividade física e saúde. Brasília, DF:Formatos Desing, 2003.60 p.

PLOWMAN, S. A.; SMITH, D. L. Fisiologia do exercício para saúde, aptidão e desempenho. Rio de Janeiro: Guanabara Koogan, 2009.

REIS FILHO, A. D. et al. Efeitos do treinamento em circuito ou caminhada após oito semanas de intervenção na composição corporal e aptidão física de mulheres obesas e sedentárias. Revista Brasileira de Obesidade, Nutrição e Emagrecimento. São Paulo, v.2, n. 11, p. 498-507. dez. 2008.

SABA, F. Mexa-se: atividade física, saúde e bem-estar. São Paulo: Takano, 2003.

FRANCISCHI, R. P.; PEREIRA, L. O.; LANCHA JUNIOR, A. H. Exercício, comportamento alimentar e obesidade: revisão dos efeitos sobre a composição corporal e parâmetros metabólico. Rev. Paul. Educ. Fís., São Paulo, v. 15, n. 2, p. 117-140, jul./dez. 2001.

Silva, D. A. S. Efeito do exercício intervalado na capacidade aeróbia, composição corporal e na população obesa: uma revisão baseada em evidências. Revista Motriz., Rio Claro, v. 16, n. 2, p. 468-476, 2010.

SOUZA, L. R., BOSSI, L. C. Treinamento resistido versus aeróbico: influência na composição corporal feminina 20-30 anos. EFDeportes.com, Revista Digital. Buenos Aires, ano. 17, n. 172, set. 2012.

WILMORE, J. H.; COSTILL, D. L. Fisiologia do esporte e do exercício. Barueri: Manole, 2010.

BARROS NETO, T. L. Fisiologia do exercício aplicada ao sistema cardiovascular. Revista da Sociedade de Cardiologia do Estado de São Paulo, São Paulo, v. 6, n. 1, p. 6-10, out.1996.

FILHO, L. A. Obesidade e Atividade Física. Jundiaí: Fontoura, 2000. 
MCARDLE, W. D.; KATCH, F. I.; KATCH, V. L. Fisiologia do exercício: energia, nutrição e desempenho humano. 7. ed. Rio de Janeiro: Guanabara Koogan, 2011.

MEDIANO, M. F. F.; GONÇALVES, T. R.; BARBOSA, J. S. O. Efeito do exercício físico sobre a composição corporal de mulheres obesas submetidas a 59 programa de perda de peso. Brazilian Journal of Biomotricity, v. 3, n. 2, p. 139-145, mar. 2009.

TROMBETTA, I. C. Exercício físico e dieta hipocalórica para o paciente obeso: vantagens e desvantagens. Rev. Bras. Hipertens, v. 10, n. 2, p. 130-133 abr./jun. 2003. 